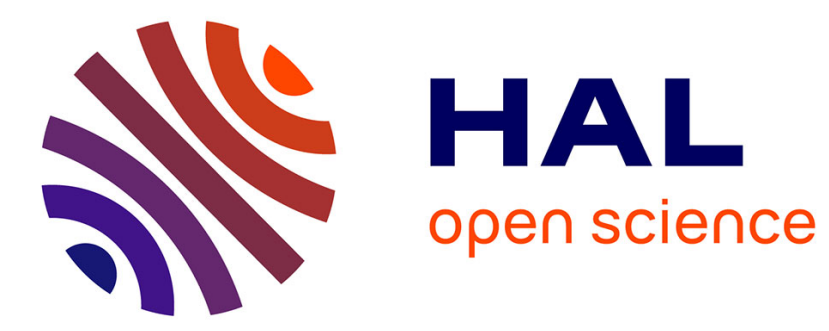

\title{
Magnetic force modelling and nonlinear switched control of an electromagnetic actuator - Extended version
}

Flavien Deschaux, Frédéric Gouaisbaut, Yassine Ariba

\section{To cite this version:}

Flavien Deschaux, Frédéric Gouaisbaut, Yassine Ariba. Magnetic force modelling and nonlinear switched control of an electromagnetic actuator - Extended version. 2019. hal-02080251v2

\section{HAL Id: hal-02080251 \\ https://hal.laas.fr/hal-02080251v2}

Preprint submitted on 4 Sep 2019

HAL is a multi-disciplinary open access archive for the deposit and dissemination of scientific research documents, whether they are published or not. The documents may come from teaching and research institutions in France or abroad, or from public or private research centers.
L'archive ouverte pluridisciplinaire HAL, est destinée au dépôt et à la diffusion de documents scientifiques de niveau recherche, publiés ou non, émanant des établissements d'enseignement et de recherche français ou étrangers, des laboratoires publics ou privés. 


\title{
Magnetic force modelling and nonlinear switched control of an electromagnetic actuator
}

\author{
Flavien Deschaux, Frederic Gouaisbaut and Yassine Ariba
}

\begin{abstract}
This paper presents the magnetic force modelling of a typical electromagnetic valve actuator system. In this work, the objective is to take into account two important features: the magnetic saturation phenomenon which is a physical problem and the positivity constraint of the magnetic force. Those issues are addressed with a switch modelling approach. The first proposed control law proves the stability in a limited set and the second one ensure the global stability of the closed loop system. For both controllers, the main part of the control consists of a two steps backstepping control, a first controller regulates the mechanical part depending on the expression of the magnetic force. And a second controller controls the coil current and the magnetic force implicitly. An illustrative example shows the effectiveness of the approach.
\end{abstract}

\section{INTRODUCTION}

For several years Electro-Magnetic Actuators (EMA) have caught much attention in the industrial activities, and especially in space [1] and automotive industries [2], [3] and [4]. In these two industrial sectors, the Electro-Magnetic Valves (EMV) are replacing the Electro-Pneumatic Valves for several factors, small sizes, an easier implementation, one needs only an electrical source and no fluid need to be present. It appears also that EMV may obtain better performances, and better reliability. That's the reason why for several years, the National Centre for Space Studies (CNES ${ }^{1}$ ) investigates innovative technologies to expand the use of EMA in Ariane and Vega launchers. The CNES has been working with CSTM, a mechanical engineering company, to replace pneumatic valves with an electromagnetic actuator [5]. The present study continues this work, in collaboration with the CNES and CSTM, and concentrate on the control issue.

EMA and EMV are usually controlled by linear control strategies such as PID [6], MPC [7] and LQR controllers [8],[1] or by nonlinear control laws like Flatness [9], Sliding mode [10], [11], [12] [13], Sontag's feedback [14] or backstepping [15].

In [16] and [1], the expression of the magnetic force has been defined as $F_{\text {mag }}=N^{2} \frac{i^{2} k_{2}}{\left(k_{0}+k_{1} x\right)^{2}} . F_{\text {mag }}$ depends on the airgap $x$ and the coil current $i . N$ and $k_{i}$ are constant parameters depending on the structure of the EMA. However,

F. Deschaux is with CNRS, LAAS,CNES, Univ de Toulouse, INSA, France, e-mail: fdeschau@laas.fr.

F. Gouaisbaut is with CNRS, LAAS, Univ de Toulouse, UPS, France, e-mail: fgouaisb@laas.fr.

Y. Ariba is with the school of engineering Icam, Toulouse, France, e-mail: yassine.ariba@icam.fr and with CNRS, LAAS, Univ de Toulouse, Toulouse, France.

${ }^{1}$ The CNES, meaning Centre National d'Etudes Spatiales, is the French space agency. this expression does not take into account some magnetic phemonon which occur in the magnetic circuit when the current increases. In order to overcome that difficulty [2] and [4] have developed a method based on magnetic force measurement to give a switched analytical expression for the magnetic force. When the current is under the saturation current, the previous nonlinear expression is proposed (called "non-saturated expression"), when the current is above the saturation current, a custom expression (called "saturated expression"), uses a polynomial fitting optimisation based on measurement.

The main objective of this paper is to consider a more realistic model of the actuator in order to take into account the magnetic phenomenon. The "non-saturated expression" was derived from a reluctance network with PLECS [17], and had been compared with finite element method simulations with COMSOL [18].The "saturated expression" was designed to fit to the experiments we made on a testbench. This approach leads to a nonlinear switched model for the magnetic force.

Two nonlinear controls have then been designed to achieve the desired closed-loop performances. The first control law, called "local controller" is based on a backstepping control. It has been developed in two steps: firstly it stabilizes the mechanical subsystem by calculating a desired magnetic force. The second step of the control drives the current in order to maintain the desired magnetic force. This control is a "local controller" because it works only in a validity region: the electromagnet can only attract the moving part of the system and the control law computes a force that repulses the moving part. So this control only works in the region where the desired computed force is positive. In some papers , [14] or [19] the solution was to send no power to the system $(u=0 V$ or bound the desired force to 0 if it is negative), but a sliding mode motion can appear and no proof of convergence is given. In [20], [15] and [21] the expression of the desired force can be negative for some condition and can induce instabilities. In this work, a second control is developed with two switching strategies that prove the global stability of the closed-loop system and avoid the problem of a negative desired force. This new control is also developped in two steps using a backstepping control: a first control is used to stabilize the position and the speed of the moving part by computing the desired force. Then a second control is used to drive the force to this latter variable. 


\section{SYSTEM DESCRIPTION AND MODELING}

\section{A. Description of the EMA}

A schematic representation of the considered EMV is shown in Fig. 1. This is a typical setup for EMV as presented in [20], [13] and [16]. The electromagnetic system is composed of an electromagnet (made with a coil winding a magnetic circuit) and a spring. The spring is used to counteract the electromagnet, which only attract the moving part and to ensure that the system returns to the open position if not powered.

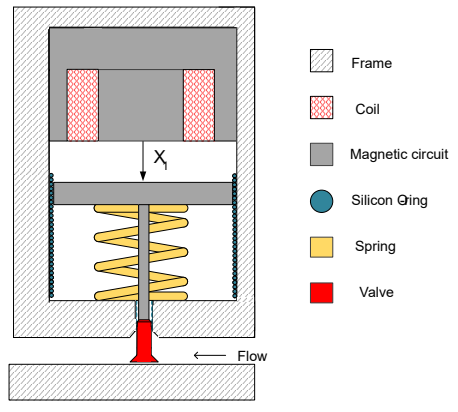

Fig. 1. Schematic of the EMA

In order to control the opening of the valve, a nonlinear stabilizing control law has been designed for this EMA. We firstly develop a nonlinear model, which takes into account the magnetic saturation by a switching expression of the magnetic force. The switching feature stems from the magnetic saturation of the circuit, when the current increases, that saturates the magnetic force. The following subsections develop the modeling steps to derive a nonlinear switched model. This EMA is modeled as a 3 dimensional system with the state vector $x=\left(x_{1}, x_{2}, x_{3}\right)^{T}$. The state components are the position $x_{1}$ of the moving part, its velocity $x_{2}$ and the current in the coil $x_{3}$. The actuator input $u$ is the voltage at the terminals of the coil.

\section{B. Experimental measurements}

The expression of the magnetic force $F_{m a g}$, the inductance $L$ and the reluctance $\rho$ defined in the control and electronic litterature [20],[6], [8] and [22]:

$$
\begin{aligned}
F_{\text {mag }}\left(x_{1}, x_{3}\right) & =\frac{1}{2} x_{3}^{2} \frac{d L\left(x_{1}\right)}{d x_{1}}, \\
L\left(x_{1}\right) & =\frac{N^{2}}{\rho\left(x_{1}\right)}, \\
\rho\left(x_{1}\right) & =\rho_{x}\left(x_{1}\right)+\rho_{0},
\end{aligned}
$$

with $N$ the number of coil's turn, $\rho_{0}$ the magnetic circuit airgap and $\rho_{x}\left(x_{1}\right)$ the airgap reluctance $\left(\neq 0 \forall x_{1}\right)$. Notice that this model has been assessed with numerical simulations in [20], using PLECS [17] and Comsol softwares [18].

Nevertheless, even if this model fits well the inductance $L$, its derivative w.r.t. $x_{1}$ is required to calculate the magnetic force $F_{m a g}$. It generally leads to inaccurate model of the force. For that reason an experimentation setup has been designed in order to measure the EMA force for given airgap and current. The schematic of the measurement bench is depicted in Fig. 2:

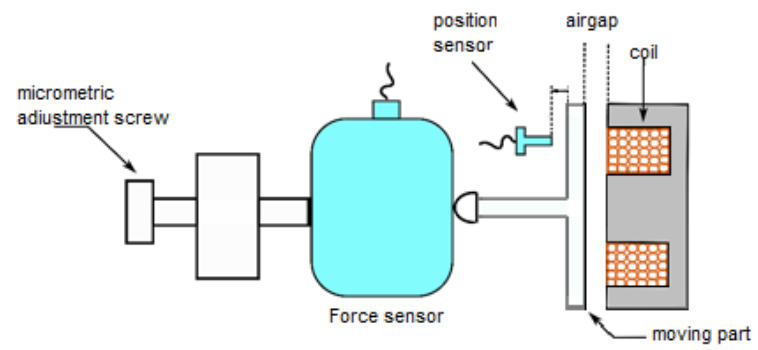

Fig. 2. Schematic of the bench for measuring the force

The Fig. 3 shows experimental measurements of the magnetic force as a function of the current and for different fixed airgaps. It shows that the proposed analytical model (1) is only valid for small values of the current. This is due to the magnetic saturation of the magnetic circuit.

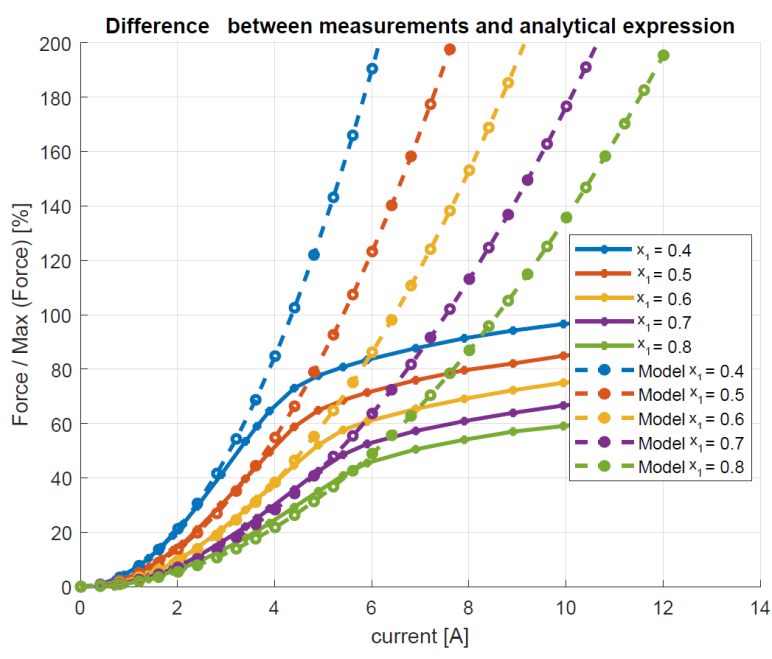

Fig. 3. Comparison between analytical model (1) and measurements

\section{Magnetic force identification}

In [4] and [2] an identification method is developped to take into account the magnetic saturation in the force modelling. This method was adapted in this paper with the experimental result of the previous paragraph. The magnetic force was split in two regions: for each airgap, it is possible to find a limit current $i_{s}$ such that, for $0<x_{3}<i_{s}$ the magnetic force can be modeled by $F_{m a g}=\frac{1}{2} x_{3}^{2} \frac{d L}{d x_{1}}$, this region is called the "non-saturated" region. For $x_{3}>i_{s}$, the equation (1) is not valid anymore, this region is called saturation region. In this latter region a parameter identification [4], gives an analytical expression of $F_{m a g}^{\text {sat }}=p_{1}\left(x_{1}\right) e^{p_{2}\left(x_{1}\right) x_{3}}+$ $p_{3}\left(x_{1}\right) e^{p_{4}\left(x_{1}\right) x_{3}}+\operatorname{cor}\left(x_{1}\right)$ where $p_{i}\left(x_{1}\right)$ are polynomials and $\operatorname{cor}\left(x_{1}\right)$ is a corrective term that ensures the continuity between both expressions of $F_{\text {mag }}$. 
Remark 1: The limit current $i_{s}$ is also identified as $i_{s}=$ $k_{6} x_{1}+k_{7}$ where $k_{6}$ and $k_{7}$ are identification constants.

\section{Magnetic force modeling}

Finally, the expression of the magnetic force is:

$$
F_{m a g}\left(x_{1}, x_{3}\right) \begin{cases}F_{m a g}^{\text {lin }} & \text { if } x_{3} \leq i_{s}\left(x_{1}\right), \\ F_{m a g}^{\text {sat }} & \text { if } x_{3}>i_{s}\left(x_{1}\right) .\end{cases}
$$

with

$$
\left\{\begin{array}{l}
F_{\text {mag }}^{\text {lin }}=\frac{1}{2} x_{3}^{2} \frac{d L}{d x_{1}} \\
F_{\text {mag }}^{\text {sat }}=p_{1}\left(x_{1}\right) e^{p_{2}\left(x_{1}\right) x_{3}}+p_{3}\left(x_{1}\right) e^{p_{4}\left(x_{1}\right) x_{3}}+\operatorname{cor}\left(x_{1}\right) .
\end{array}\right.
$$

The results of the force computation is depicted in Fig.

4. The originality of the model is to use a switched state-

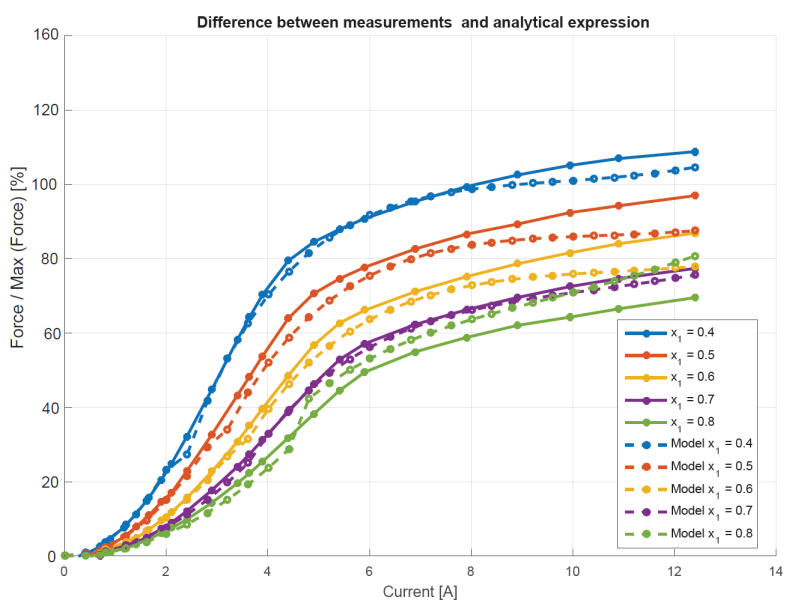

Fig. 4. Comparaison between analytical model (2) and measurements

dependent expression for the expression of $F_{\text {mag }}$ and a new expression of the inductance $L$. These expressions take into account two magnetic phenomenon arisen in the magnetic circuit called flux fringing (it affects $\rho$ in equation (1)) and magnetic saturation (it affects the equations (2)). A previous work on the flux fringing was done in [20].

\section{E. Magnetic force properties}

Due to the particular form of the magnetic force $F_{m a g}$ in equation (2), it is important to emphasize some properties that will be considered in the control design.

Property 1: $\forall\left(x_{1}, x_{3}\right) \in \mathbb{R}^{2}, F_{\text {mag }}\left(x_{1}, x_{3}\right) \geq 0$.

Property 2: $\forall\left(x_{1}, x_{3}\right) \in \mathbb{R}^{2}$, the function $F_{\text {mag }}\left(x_{1}, x_{3}\right)$ is a continous and bounded function.

Property 3: For a fixed $x_{1} \in \mathbb{R}, \forall x_{3} \in \mathbb{R}, F_{\text {mag }_{1}}\left(x_{3}\right)$ is monotonically increasing.

Property 4: $\forall F_{d}>0, \forall x_{1}, \exists ! x_{3}, F_{m a g}\left(x_{1}, x_{3}\right)=F_{d}$

Proof: By using the properties 2 and 3, for a fixed $x_{1}$ means that $F_{\text {mag }_{x_{1}}}\left(x_{3}\right)$ is bijective. So it is possible to find an application $F_{m a g}^{-1}$ such that $F_{m a g}^{-1}\left(x_{1}, F_{d}\right)=x_{3}$

\section{F. Electrical part}

Applying an input voltage $u$ at the terminals of the coil the electrical dynamic is described by [20] [22]:

$$
u=R x_{3}+L\left(x_{1}\right) \frac{d x_{3}}{d t}+x_{3} \frac{d L\left(x_{1}\right)}{d t},
$$

with $R$ the coil internal resistance. A dynamical equation for the current $x_{3}$ is formulated as

$$
\frac{d x_{3}}{d t}=\frac{1}{L\left(x_{1}\right)}\left(u-R x_{3}+x_{2} x_{3} \frac{\partial L\left(x_{1}\right)}{\partial x_{1}}\right) .
$$

\section{G. Mechanical part}

The application of the Newton's second law to the moving part gives:

$$
m \frac{d x_{2}}{d t}=-F_{m a g}+F_{\text {ext }},
$$

where $F_{\text {ext }}$ is the sum of the external forces: $F_{\text {ext }}=F_{\text {friction }}+$ $F_{\text {spring. }} F_{\text {friction }}$ represents the friction force which is proportional to the speed, $F_{\text {friction }}=-\lambda x_{2}, \lambda>0 . F_{\text {spring }}$ is the force due to the spring, proportional to the position, $F_{\text {spring }}=-K\left(x_{1}-x_{0}\right), K>0$ and $x_{0}$ the spring free length.

\section{H. State space model}

The state variables have been defined in Paragraph II-A, and gathering equations (5) and (6), a state space model is obtained in (7). Note that the control input $u(t)$ has effect only on the third equation. Let $x_{i n i}$ be the initial state of the actuator.

$$
\left\{\begin{array}{l}
\dot{x}_{1}=x_{2}, \\
\dot{x}_{2}=\frac{1}{m}\left[-F_{\text {mag }}\left(x_{1}, x_{3}\right)-\lambda x_{2}-K\left(x_{1}-x_{0}\right)\right], \\
\dot{x}_{3}=\frac{1}{L\left(x_{1}\right)}\left[u-R x_{3}+x_{2} x_{3} \frac{\partial L}{\partial x_{1}}\right] .
\end{array}\right.
$$

In the two following parts, two types of controllers have been developed. Both methods proposed use a backstepping control which takes into account the switched model of the force and the positivity constraint. These controllers make the closed loop system states converge to the desired equilibrium point, which depends on the position reference signal $y_{r}$ the state $x_{1}$ must track.

\section{LOCAL BACKSTEPPING CONTROL}

In this first subsection, a local backstepping control is designed to control the EMA. This control reveals itslef local since it is only valid in the region for which $F_{m a g} \geq 0$, called validity region.

1) Control of the mechanical subsystem: The controller design starts by the control of the position and velocity states. The choice of the backstepping method is natural due to the cascade form of the subsystem (8),

$$
\left\{\begin{array}{l}
\dot{x}_{1}=x_{2}, \\
\dot{x}_{2}=\frac{1}{m}\left[-F_{m a g}\left(x_{1}, x_{3}\right)-\lambda x_{2}-K\left(x_{1}-x_{0}\right)\right] .
\end{array}\right.
$$


We aim at finding the desired current $x_{3 d}$ that stabilises the subsystem (8) to the equilibrium point $\left[y_{r} ; 0\right]^{T}$. As it may be complicated to express the desired current $x_{3 d}$ due to the switched expression of $F_{\text {mag }}$, it is more convenient to find the desired magnetic force $F_{d}$ to stabilize this susbsytem.

Theorem 1: Consider $\alpha_{1}, \alpha_{2}$ two positives scalars, the virtual control law $F_{d}=m\left(a z_{1}+\left(b+\alpha_{2}\right) z_{2}-d\right)$ with $a=$ $1-\alpha_{1}^{2}+\frac{\lambda}{m} \alpha_{1}-\frac{K}{m}, b=\alpha_{1}-\frac{\lambda}{m}$ and $d=\frac{K}{m}\left(y_{r}-x_{0}\right)$. makes the subsystem (8) converge to $\left(y_{r}, 0\right)$.

Proof: Consider the classical change of variable

$$
\left\{\begin{array}{l}
z_{1}=x_{1}-y_{r} \\
z_{2}=x_{2}+\alpha_{1} z_{1}
\end{array}\right.
$$

with $\alpha_{1}$ a positive scalar. The subsystem (8) can be rewritten as:

$$
\left\{\begin{aligned}
\dot{z}_{1} & =-\alpha_{1} z_{1}+z_{2} \\
\dot{z}_{2} & =\frac{1}{m}\left[-F_{d}-\lambda\left(z_{2}-\alpha_{1} z_{1}\right)-K\left(z_{1}+y_{r}-x_{0}\right)\right] \\
& +\alpha_{1} z_{2}-\alpha_{1}^{2} z_{1}
\end{aligned}\right.
$$

In order to achieve the desired closed-loop properties, let us consider a Lyapunov function of the form:

$$
V_{1}=\frac{1}{2} z_{1}^{2}+\frac{1}{2} z_{2}^{2}
$$

The derivative of $V_{1}$ along the trajectories of (10) leads to:

$$
\dot{V}_{1}=-\alpha_{1} z_{1}^{2}+a z_{1} z_{2}+b z_{2}^{2}-z_{2} d-z_{2} \frac{1}{m} F_{d}
$$

with $a=1-\alpha_{1}^{2}+\frac{\lambda}{m} \alpha_{1}-\frac{K}{m}, b=\alpha_{1}-\frac{\lambda}{m}$ and $d=\frac{K}{m}\left(y_{r}-x_{0}\right)$. Consider the control law $F_{d}=m\left(a z_{1}+\left(b+\alpha_{2}\right) z_{2}-d\right)$ where $\alpha_{2}$ is a positive scalar. $\dot{V}_{1}$ can then be rewritten as:

$$
\dot{V}_{1}=-\alpha_{1} z_{1}^{2}-\alpha_{2} z_{2}^{2} \leq 0 \quad \forall z_{1}, z_{2}
$$

Remark 2: The dynamic of the closed loop system (10) is linear with the control of theorem (1) :

$$
\left(\begin{array}{l}
\dot{z}_{1} \\
\dot{z}_{2}
\end{array}\right)=\left(\begin{array}{cc}
-\alpha_{1} & 1 \\
-1 & -\alpha_{2}
\end{array}\right)\left(\begin{array}{l}
z_{1} \\
z_{2}
\end{array}\right)
$$

and the choice of gains $\alpha_{1}$ and $\alpha_{2}$ adjusts the convergence speed.

Remark 3: Notice that, by construction $F_{\text {mag }}$ is always positive, due to property 1 , while the expression of the desired force $F_{d}$ may be not always positive.

In the next subsection, we aim at finding an estimation of the region for which the calculated magnetic force is positive. This estimation is called validity region.
2) Estimation of the validity region:

lemma 1: An estimation of the validity region is the largest level line of $V\left(z_{1}, z_{2}\right)$ where there is a single intersection point between $V\left(z_{1}, z_{2}\right)$ and $F_{d}\left(z_{1}, z_{2}\right)=0$. This set is defined as: $\exists C \in \mathbb{R}^{+}$such that $\mathbb{D}=\left\{\left(z_{1}, z_{2}\right) \in \mathbb{R}^{2} \mid V\left(z_{1}, z_{2}\right) \leq\right.$ $C\}$.

Proof: $\mathbb{D}$ is an invariant set for the closed-loop subsystem. Starting from $\mathbb{D}$ induces that the state will remain in $\mathbb{D}$ and therefore converges to the equilibrium point $\left[y_{r} ; 0\right]^{T}$. Moreover, if all the initials conditions of the EMA are in the estimated validity region then the desired force $F_{d}$ will remain non negative.

The Fig 5 shows the estimated validity region.

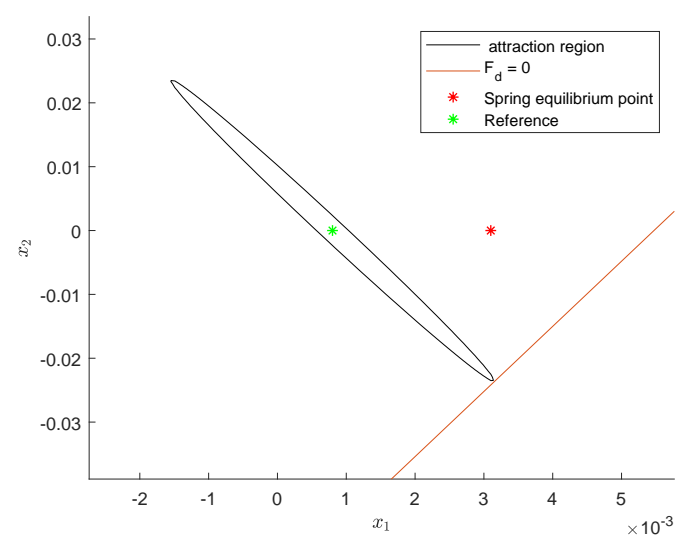

Fig. 5. Approximation of the validity region for $\alpha_{1}=\alpha_{2}=100$

3) Design of the local EMA controller:

The full system (15) is now considered:

$$
\left\{\begin{aligned}
\dot{z}_{1} & =-\alpha_{1} z_{1}+z_{2} \\
\dot{z}_{2} & =\frac{1}{m}\left[-F_{m a g}\left(z_{1}, x_{3}\right)-\lambda\left(z_{2}-\alpha_{1} z_{1}\right)-K\left(z_{1}+y_{r}-x_{0}\right)\right] \\
& +\alpha_{1} z_{2}-\alpha_{1}^{2} z_{1} . \\
\dot{x}_{3} & =\frac{1}{L\left(z_{1}\right)}\left[u-R x_{3}+\left(z_{2}-\alpha_{1} z_{1}\right) x_{3} \frac{\partial L}{\partial z_{1}}\right] \\
& =f_{x_{3}}\left(z_{1}, z_{2}, x_{3}\right)+g_{x_{3}}\left(z_{1}, z_{2}, x_{3}\right) u .
\end{aligned}\right.
$$

Theorem 2: Assume the initial condition $x_{\text {ini }} \in \mathbb{D}$, then the control

$$
u=\frac{1}{g_{F}(z)}\left[-\alpha_{3}\left(F_{m a g}-F_{d}\right)+\frac{z_{2}}{m}+\dot{F}_{d}-f_{F}(z)\right],
$$

with $\alpha_{3}>0, z=\left(z_{1}, z_{2}, x_{3}\right)$ and $\dot{F}_{m a g}=f_{F}(z)+g_{F}(z) u$ where

$$
f_{F}(z)= \begin{cases}f_{x_{3}}(z) x_{3} \frac{d L}{d z_{1}}+\frac{1}{2} x_{3}^{2}\left(\frac{\dot{d} L}{d z_{1}}\right) & \text { if } x_{3} \leq i_{s} \\ \dot{p}_{1} e^{p_{2} x_{3}}+\dot{p}_{3} e^{p_{4} x_{3}}+c \dot{o} r & \\ +\left(p_{1} p_{2} e^{p_{2} x_{3}}+p_{3} p_{4} e^{p_{4} x_{3}}\right) f_{x_{3}}(z) & \\ +p_{1} \dot{p}_{2} x_{3} e^{p_{2} x_{3}}+p_{3} \dot{p}_{4} x_{3} e^{p_{4} x_{3}} & \text { if } x_{3}>i_{s}\end{cases}
$$

and

$$
g_{F}(z)= \begin{cases}g_{x_{3}}(z) x_{3} \frac{d L}{d z_{1}} & \text { if } x_{3} \leq i_{s} \\ \left(p_{1} p_{2} e^{p_{2} x_{3}}+p_{3} p_{4} e^{p_{4} x_{3}}\right) g_{x_{3}}(z) & \text { if } x_{3}>i_{s}\end{cases}
$$


makes the system (15) stable and makes the position converges to $y_{r}$.

Proof: Consider the following Lyapunov function candidate $V=V_{1}\left(z_{1}, z_{2}\right)+\frac{1}{2}\left(F_{\text {mag }}\left(z_{1}, x_{3}\right)-F_{d}\left(z_{1}, z_{2}\right)\right)^{2}$. The calculation of the desired equilibrium point of the system (15) gives the equilibrium force desired $F_{d}=-K\left(z_{1}+y_{r}-x_{0}\right)$. In other words, the electromagnet has to compensate the spring at the equilibrium. So it is possible to find the equilibrium current $x_{3 e q}=F_{m a g}^{-1}\left(y_{r}, K\left(y_{r}-x_{0}\right)\right)$. $V$ is a Lyapunov function candidate in $\left(z_{1}, z_{2}, x_{3}\right)$ since $V\left(z^{*}\right)=0$ with $z^{*}=\left(0,0, x_{3 e q}\right)$ Let us derive $V$ along the trajectories of (15)

$$
\begin{aligned}
\dot{V}= & -\alpha_{1} z_{1}^{2}+a z_{1} z_{2}+b z_{2}^{2}-d z_{2}-\frac{z_{2}}{m} F_{m a g} \\
& +\left(\dot{F}_{m a g}-\dot{F}_{d}\right)\left(F_{m a g}-F_{d}\right) \\
= & -\alpha_{1} z_{1}^{2}+a z_{1} z_{2}+b z_{2}^{2}-d z_{2}-\frac{z_{2}}{m} F_{d}-\frac{z_{2}}{m}\left(F_{m a g}-F_{d}\right) \\
& +\left(\dot{F}_{m a g}-\dot{F}_{d}\right)\left(F_{m a g}-F_{d}\right) \\
= & -\alpha_{1} z_{1}^{2}-\alpha_{2} z_{2}^{2}-\frac{z_{2}}{m}\left(F_{m a g}-F_{d}\right) \\
& +\left(\dot{F}_{m a g}-\dot{F}_{d}\right)\left(F_{m a g}-F_{d}\right) .
\end{aligned}
$$

If $\dot{F}_{m a g}=-\alpha_{3}\left(F_{m a g}-F_{d}\right)+\frac{z_{2}}{m}+\dot{F}_{d}$, with $\alpha_{3}>0$ then

$$
\dot{V}=-\alpha_{1} z_{1}^{2}-\alpha_{2} z_{2}^{2}-\alpha_{3}\left(F_{m a g}-F_{d}\right)^{2}
$$

with

$u=\frac{1}{g_{F}\left(z_{1}, z_{2}, x_{3}\right)}\left[-\alpha_{3}\left(F_{m a g}-F_{d}\right)+\frac{z_{2}}{m}+\dot{F}_{d}-f_{F}\left(z_{1}, z_{2}, x_{3}\right)\right]$.

We obtain the desired value of $\dot{F}_{\text {mag }}$ because it can be expressed as $\dot{F}_{\text {mag }}=f_{F}\left(z_{1}, z_{2}, x_{3}\right)+g_{F}\left(z_{1}, z_{2}, x_{3}\right) u$. Finally, $\exists \alpha=\max \left(\alpha_{i}\right)$ such that $\dot{V} \leq-\alpha V$

$$
\Rightarrow V(t)<V(0) e^{-\alpha t} \Leftrightarrow\left\{\begin{array}{rl}
z_{1} & =0 \\
z_{2} & =0 \\
F_{\text {mag }} & =F_{d}
\end{array} \Rightarrow x_{3}=x_{3 d} .\right.
$$

Hence, the input variable $u$ controls the system through the derivative of the magnetic force. That concludes the proof.

\section{GLOBAL BACKSTEPPING CONTROL}

The previous controller is local because there is no proof of convergence if the states are not in the validity region. Besides, the control law (19) may be not feasible in practice if $F_{d}$ becomes negative. The following switching strategies can solve this problem by proving the global asymptotic stability of the closed loop system. The main idea is to find a control that makes the state convergent to a fixed point in $\mathbb{D}$ when the state is not in $\mathbb{D}$. The strategies use the spring property: if $u=0$, the state converges to the spring equilibrium point $X 0=\left(x_{0}, 0\right)$ but actually this equilibrium point is not in $\mathbb{D}$ as shown in Fig. 5 .

\section{A. Region of validity optimization}

We aim at including the equilibrium point of the spring $X 0=\left(x_{0}, 0\right)$ into $\mathbb{D}$ by using the control define in Theorem 1: a virtual control law $F_{d}=m\left(-\alpha_{1} z_{1}-\alpha_{2} z_{2}-d\right)$, with $\alpha_{1}$, $\alpha_{2}$ two positives scalars, stabilize the subsystem (10) for any initial conditions in $\mathbb{D}$. In order to optimize the size of the domain $\mathbb{D}$, let consider a more general Lyapunov function candidate.

$$
V=Z^{T} P Z
$$

with $Z=\left(z_{1}, z_{2}\right)$ and $P$ a definite positive matrix.

Theorem 3: If $F_{d}=-\frac{K}{m}\left(y_{r}-x_{0}\right)-\alpha_{1} m z_{1}-\alpha_{2} m z_{2}, \exists P>$ $0, \exists Q>0$ such that $\dot{V}=A^{T} P+P A<-Q$ and $\dot{V}<-\alpha V$ with $\alpha>0$ and $V=Z^{T} P Z$.

Proof: The derivative of $V$ along the trajectories gives

$$
\begin{aligned}
\dot{V} & =a_{1} z_{1}^{2}+a_{2} z_{2}^{2}+a_{3} z_{1} z_{2}+a_{4} \frac{K}{m}\left(y_{r}-x_{0}\right) z_{1} \\
& +a_{5} \frac{K}{m}\left(y_{r}-x_{0}\right) z_{2}-F_{d}\left(-a_{4} z_{1}-a_{5} z_{2}\right) .
\end{aligned}
$$

If $F_{d}=-\frac{K}{m}\left(y_{r}-x_{0}\right)-\alpha_{1} m z_{1}-\alpha_{2} m z_{2}$, then the dynamic of $\left(z_{1}, z_{2}\right)$ becomes

$$
\begin{gathered}
\left(\begin{array}{c}
\dot{z}_{1} \\
\dot{z}_{2}
\end{array}\right)=\left(\begin{array}{cc}
-\alpha_{1} & 1 \\
\alpha_{1}-\alpha_{1}^{2}+\alpha_{1} \lambda / m-K / m & \alpha_{1}+\alpha_{2}-\lambda / m
\end{array}\right)\left(\begin{array}{c}
z_{1} \\
z_{2}
\end{array}\right) \\
\left(\begin{array}{c}
\dot{z}_{1} \\
\dot{z}_{2}
\end{array}\right)=A\left(\begin{array}{l}
z_{1} \\
z_{2}
\end{array}\right) \text { with } A \text { a stable matrix. }
\end{gathered}
$$

And the equation (22) becomes:

$$
\begin{aligned}
\dot{V} & =\left(a_{1}-\alpha_{1} m a_{4}\right) z_{1}^{2}+\left(a_{2}-\alpha_{2} m a_{5}\right) z_{2}^{2} \\
& +\left(a_{3}-\alpha_{1} m a_{5}-\alpha_{2} m a_{4}\right) z_{1} z_{2}, \\
\dot{V} & =a_{1}^{\prime} z_{1}^{2}+a_{2}^{\prime} z_{2}^{2}+a_{3}^{\prime} z_{1} z_{2}, \\
\dot{V} & =-Z^{T}\left(A^{T} P+P A\right) Z, \\
\dot{V} & =-Z^{T} Q Z,
\end{aligned}
$$

where $P$ is positive definite by definition and as $A$ is stable it means that $Q$ is a positive definite matrix. So the following $L M I$ procedure is used to find a $P$ matrix under the following constraints [23], [24].

objective function Min $\operatorname{tr}(P)$

under constraints $A^{T} P+P A<-\alpha P$

For a fixed $\alpha$, the LMI solver is able to find a feasible $P$ matrix. So equation (25) becomes

$$
\begin{aligned}
& \dot{V}<-Z^{T} \alpha P Z \\
& \dot{V}<-\alpha V
\end{aligned}
$$

By minimizing $\operatorname{tr}(P)$, we minimize the eigenvalues of $P$ and the constraints allow to find only positive eigenvalues, which concludes the proof.

Remark 4: This LMI is solved only once time offline in order to give a better estimation of the validity region $\mathbb{D}$ by using a new Lyapunov function (with a fixed control $F_{d}$ ). The parameter $\alpha$ is arbitrarily chosen to include the spring equilibrium point $X 0$ in $\mathbb{D}$. And the gains $\alpha_{i}$ are design to respect the required closed-loop performances.

The Fig. 6 shows the expansion of the validity region. The spring equilibrium point $X 0$ is now included in $\mathbb{D}$

The following two subsection will develop two control strategies in order to prove the global stability of the closed loop system. 


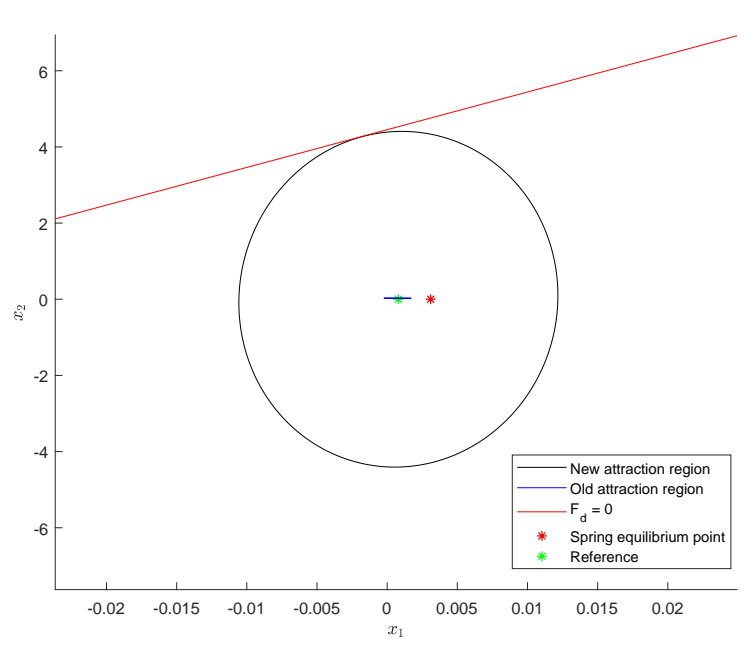

Fig. 6. validity region optimisation

\section{B. Intuitive switching control strategy}

If the desired force $F_{d}$ is negative, the best answer is to force $F_{d}$ to zero. Because when the system is not controlled $(u=0)$, it converges asymptotically to the equilibrium point $X 0$ of the spring, because it is a mass-spring system and $X 0 \in \mathbb{D}$. And finally, if $x(t) \in \mathbb{D}$, the control (19) makes the equilibrium point $\left[y_{r}, 0\right]^{T}$ stable and attractive.

This first switching strategy is explained with the following two cases:

- case $1: x(t) \in \mathbb{D}$

The control $u$ of theorem 1 and $F_{d}=$ $m\left(-\alpha_{1} z_{1}-\alpha_{2} z_{2}-d\right)$ are choosen and the system converges to the desired equilibrium point $y_{r}$

- case 2: $x(t) \in \overline{\mathbb{D}}$

The control $u=0$ is enforced and there is a time where the trajectorie $x(t)$ hits $\mathbb{D}$ because $X 0$ is attractive and it returns to the case 1 .

The case 1 is already proven in Theorem 1 . For the case 2 , let prove that $x(t)$ passes from $V(x)=c$ to $V(x)=c_{1}$, $c>c_{1}>0$ in finite time

lemma 2: If $V(x)$ is a Lyapunov function for $x(t)$, all the trajectories $x(t) \in \mathbb{R}^{3}$ passes from $V(x)=c$ to $V(x)=c_{1}$, $c>c_{1}>0$ in finite time

Proof: $\exists c>0, \varepsilon>0$ such that the sets $\{V(x) \leq c\}$ and $\Lambda=\{\varepsilon \leq V(x) \leq c\}$ are compacts and $\mathbb{D} \subset \Lambda . \exists W(x)$ a continous function positive definite such that $\dot{V}(x) \leq-W(x)$ $\forall x \in \Lambda \forall t>t_{0}$ choose $k=\min _{x \in \Lambda} W(x), k$ is positive and exists because $W(x)$ is continous and $\Lambda$ is compact. It leads to

$$
\begin{aligned}
W(x) & \geq k & & \forall x \in \Lambda \\
\dot{V}(x(t)) & \leq-k & & \forall x \in \Lambda, \forall t>t_{0} \\
V(x(t)) & \leq V\left(x\left(t_{0}\right)\right)-k\left(t-t_{0}\right) & & \forall x \in \Lambda, \forall t>t_{0} \\
V(x(t)) & \leq c-k\left(t-t_{0}\right) & & \forall x \in \Lambda, \forall t>t_{0}
\end{aligned}
$$

It means that $V(x(t))<c_{1}$ in the time interval $\left[t_{0}, t_{0}+\frac{c-c_{1}}{k}\right]$ where $t_{0}$ is the initial time.

Remark 5: With this control strategy, no sliding mode motion can occured on $F_{d}=0$ because at most, it switches once: it occurs when the initial conditions $x_{\text {ini }} \notin \mathbb{D}$, then once in $\mathbb{D}$, the trajectory remains in $\mathbb{D}$. The full system (15) is now considered.

Theorem 4: If the validity region $\mathbb{D}$ contains the spring equilibrium point $X 0$ the following switched control law makes the system (15) convergent to $\left(y_{r}, 0, x_{3 e q}\right)$ : Consider $\alpha_{1}, \alpha_{2}, \alpha_{3}$ three positives scalars, $z_{3}=F_{m a g}-F_{d}$ and $z=$ $\left(z_{1}, z_{2}, z_{3}\right)$ the error state vector.

$$
u= \begin{cases}0 & ; \text { if } z(t) \notin \mathbb{D} \\ \frac{1}{g_{F}(z)}\left[-\alpha_{3} z_{3}+\frac{z_{2}}{m}+\dot{F}_{d}-f_{F}(z)\right] & ; \text { else }\end{cases}
$$

with $d=\frac{K}{m}\left(y_{r}-x_{0}\right), F_{d}=m\left(-\alpha_{1} z_{1}-\alpha_{2} z_{2}-d\right)$ and $z(t)$ the trajectories of the errors states.

Proof: The proof is given by following those of Theorem 1 and lemma 2.

case $1:$ In case of $x_{i n i} \in \mathbb{D}$ the derivative of the Lyapunov function $V=V_{1}\left(z_{1}, z_{2}\right)+\frac{1}{2} z_{3}^{2}$ along the trajectories with $z_{3}=$ $F_{\text {mag }}-F_{d}$ leads to:

$$
\begin{aligned}
\dot{V} & =-\alpha_{1} z_{1}^{2}+a z_{1} z_{2}+b z_{2}^{2}-d z_{2}-\frac{z_{2}}{m} F_{m a g}+\left(\dot{F}_{m a g}-\dot{F}_{d}\right) z_{3} \\
& =-\alpha_{1} z_{1}^{2}-\alpha_{2} z_{2}^{2}-\alpha_{3} z_{3}^{2}
\end{aligned}
$$

case $2:$ In case of $x_{i n i} \in \overline{\mathbb{D}}$, as the control $u$ is set to zero, it leads to a mass-spring system which converges to the spring equilibrium point $X 0$. But remembering that $X 0 \in \mathbb{D}$, by lemma $2, \exists t_{1}>t_{0}$ where $x\left(t_{1}\right) \in \mathbb{D}$, the switching control leads to case 1 and $\forall t>t_{1}$,

$$
\dot{V}=-\alpha_{1} z_{1}^{2}-\alpha_{2} z_{2}^{2}-\alpha_{3} z_{3}^{2}
$$

which concludes the proof

Remark 6: This strategy is "intuitive" because we only have an action on the system in $\mathbb{D}$ which is a restricted set of the space. We essentially use the physical properties of the spring to ensure the global asymptotic stability. The next control law aims at enlarging the set where the control (28)is enabled.

\section{Hybrid switching control strategy}

If the region of validity $\mathbb{D}$ contains the equilibrium point $X 0$, the following result develop a switching strategy in order to prove the global stability. The switching strategy is explained with the following 3 cases:

- case $1: x_{\text {ini }} \in \mathbb{D}$ The control $u$ of Theorem 1 and $F_{d}=m\left(-\alpha_{1} z_{1}-\alpha_{2} z_{2}-d\right)$ are choosen and the system converges to the desired equilibrium point $\left[y_{r}, 0\right]^{T}$

- case 2: $x_{i n i} \in\left\{F_{d}<0\right\}$ The control $u=0$ is choosen and as the spring equilibrium point $X 0 \in \mathbb{D}$, there exists $t_{1}>t_{0}$ where $x\left(t_{1}\right) \in \mathbb{D}$. Then when $x(t) \in \mathbb{D}$, the control of case 1 is enabled. 
- case 3: $x_{\text {ini }} \in\left\{F_{d}>0\right\} \cap \overline{\mathbb{D}}$

The control $u$ of Theorem 1 and $F_{d}=$ $m\left(-\alpha_{1} z_{1}-\alpha_{2} z_{2}-d\right)$ are choosen and the trajectories $x(t)$ have two options : $F_{d}$ remains positive and there is an instant where the trajectories $x(t)$ enter in $\mathbb{D}$ and then the control of case 1 is enabled. Or, there is an instant where $x(t)$ enter in $\left\{F_{d}=0\right\}$ and then the control of case 2 is enabled.

The case 1 is already proven in Theorem 4 . For the cases 2 and 3, the use of lemma 2 proves that $x\left(t_{0}\right)$ passes from $V\left(x\left(t_{0}\right)\right)=c$ to $V\left(x\left(t_{1}\right)\right)=c_{1} \subset \mathbb{D}, c>c_{1}>0$ in a finite time $t_{1}$.

Remark 7: As the previous control strategy, there is no sliding on $F_{d}=0$ because at most, it switches twice: it occurs when the initial conditions $x_{i n i} \notin \mathbb{D}, F_{d}\left(x_{i n i}\right)>0$ and when the trajectories lead in a finite time $t_{1}$ to $F_{d}\left(x\left(t_{1}\right)\right)<0$.

Remark 8: This control strategy is an hybrid control and can be modeled in the framework proposed by [25] using a token $M$ to take into account the fact that the trajectory $x(t)$ has ever been in region $\left\{F_{d}<0\right\}$.

So let consider the flow set

$$
\mathbb{F}_{f}:=\left\{\{M=1\} \times\left\{F_{d} \geq 0\right\} \text { or }\{M=0\} \times\{z \mid V(z) \leq C\}\right\} .
$$

Let consider the jump set

$\mathbb{D}_{f}:=\left\{\{M=1\} \times\left\{F_{d} \leq 0\right\}\right.$ or $\left.\{M=0\} \times\{z \mid V(z)>C\}\right\}$.

The closed loop system can be rewritten as

$$
\left\{\begin{aligned}
\dot{z} & =f\left(z(t), u_{M}(z)\right) \\
\dot{M} & =0
\end{aligned}\right\} \text { if }(z, M) \in \mathbb{F}_{f}
$$

where

$$
u=\left\{\begin{array}{l}
u_{1}(z)=\frac{1}{g_{F}(z)}\left[-\alpha_{3} z_{3}+\frac{z_{2}}{m}+\dot{F}_{d}-f_{F}(z)\right] \\
u_{0}(z)=0
\end{array}\right.
$$

with $d=\frac{K}{m}\left(y_{r}-x_{0}\right), F_{d}=m\left(-\alpha_{1} z_{1}-\alpha_{2} z_{2}-d\right), z(t)$ the trajectories of the errors states, and $M\left(t_{0}\right)=1$. Inspired by [25], the closed loop system satisfies the standard conditions which ensures the well-posedness of the closed-loop system.

Theorem 5: Assume the closed-loop system (33), and consider the compact set $\mathbb{A}=\left\{x=x_{e q}, M \in\{0,1\}\right\}$ then $\mathbb{A}$ is globaly asymptotically stable.

Proof: The proof is made with two steps. Firstly, $\mathbb{A}$ is stable by construction following Theorem 4. Secondly $\mathbb{A}$ is attractive and the lemma 2 ensures that in the worst case $\exists t_{2}>t_{0}$ where $x\left(t_{2}\right) \in\left\{F_{d}<0\right\}$ and $\exists t_{3}>t_{2}$ where $x\left(t_{3}\right) \in$ $\mathbb{D}$. Finally, the closed-loop system is globaly asymptotically stable which concludes the proof.

\section{Simulation}

The value of the EMA parameters is defined in [20]. The results of these switched control are shown for different inital conditions (CI) in Fig. 7 in the plan $\left(x_{1}, x_{2}\right)$. For the two last initial conditions $\mathrm{n}^{\mathrm{O}} 2$ and $\mathrm{n}^{\circ} 3$, the result of the hybrid control and the intuitive control are really close. For the first initial condition, the convergence time is slightly larger for the hybrid control than the intuitive control. In this case, the initial position is over the desired position, so to comeback to the desired position $F_{\text {mag }}$ has to be lower than the spring strenght. With the intuitive control $F_{\text {mag }}=0$ and with the hybrid control $0<F_{\text {mag }}<F_{\text {spring }}$ so it is slower in this case.

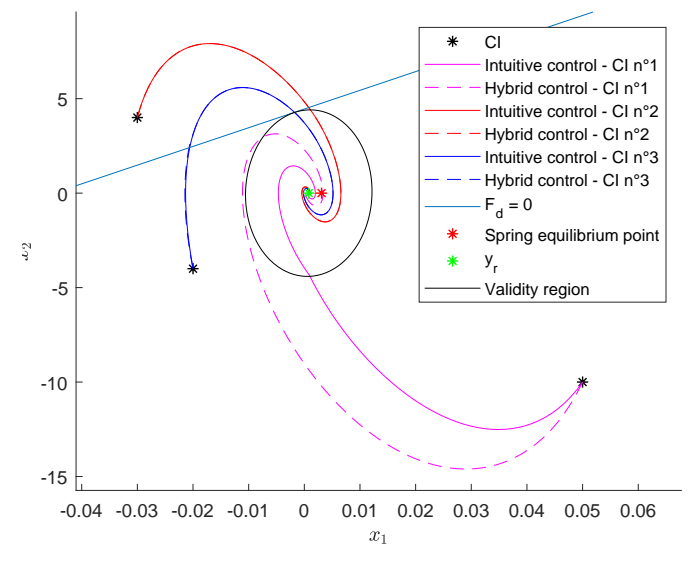

Fig. 7. Dynamics of the controlled subsystem

The speed of convergence of the Lyapunov functions are shown for different inital conditions in Fig. 8.

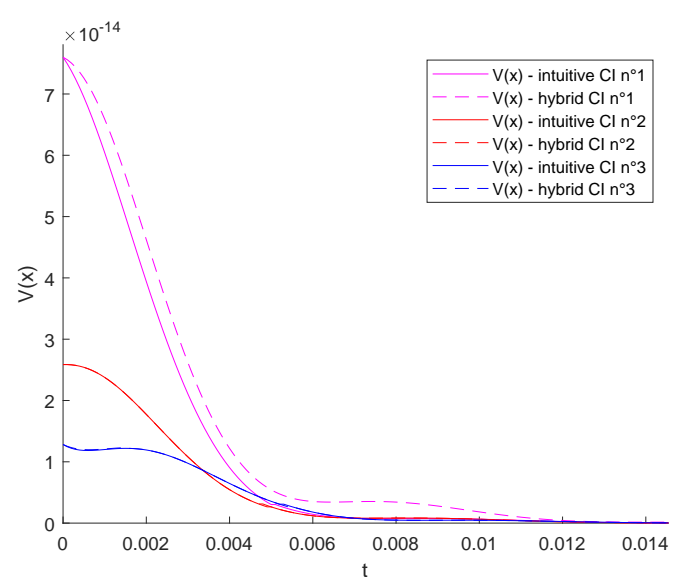

Fig. 8. Values of the Lyapunov function for the differents control laws

A position tracking simulation was computed, with $y_{r}$ a periodic square signal. The results of the position tracking simulation are shown in the Fig.9. The speed and the current are respectively depicted in Fig.10 and Fig.11. 


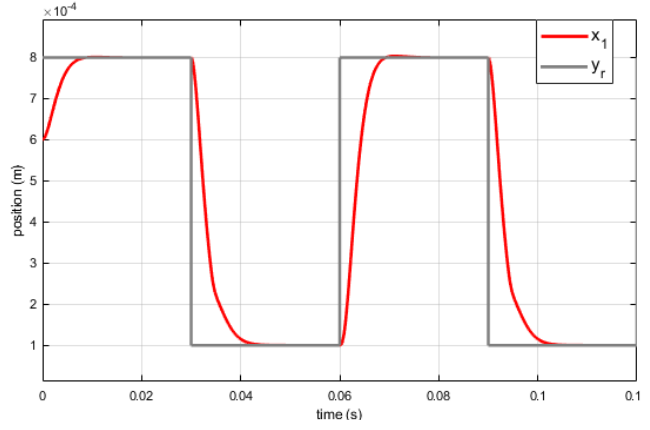

Fig. 9. Simulation of the position tracking

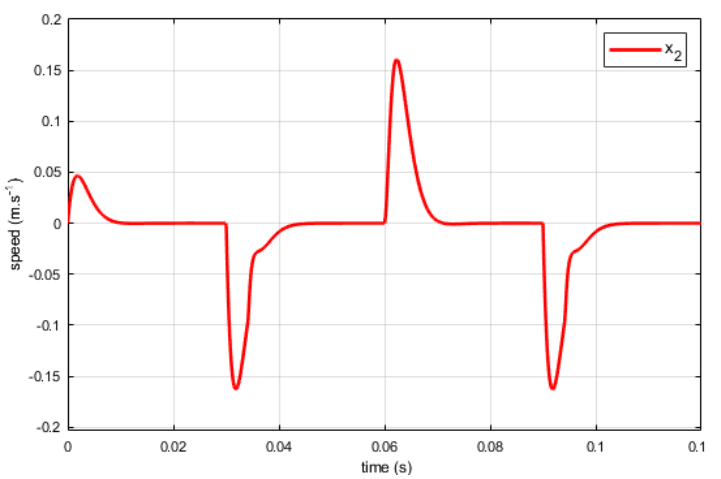

Fig. 10. Simulation of the speed evolution during the position traking

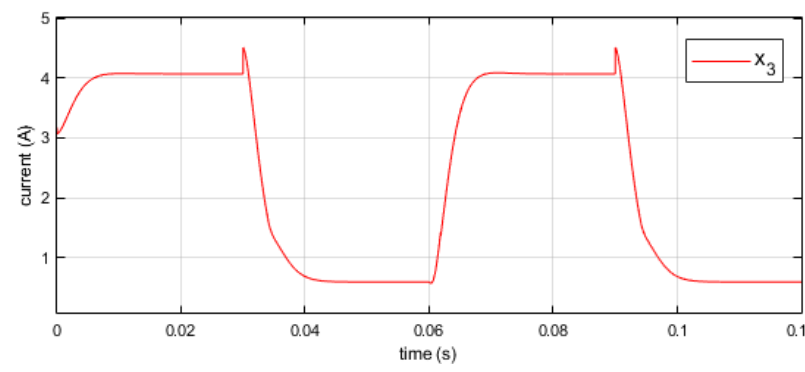

Fig. 11. Simulation of the current evolution during the position traking

\section{CONCLUSION}

This paper focuses on the control law design for an EMA which takes into account the magnetic saturation and the positivity constraint of the magnetic force. The two lasts proposed controls laws relies on a backstepping control with two different switching strategies that ensure the global asymptotic stability of the closed-loop system. An interesting extension of this work will consist in taking a more generic Lyapunov function for the local controller. It will change the expression of the desired force $F_{d}$ and it may enlarge the validity region $\mathbb{D}$.

Future work consists in validating the control law on the testbed we developed.

\section{ACKNOWLEDGMENT}

The Authors would like to thank Damien De Seze from CNES and François Dugué from CSTM for the grants that partly supports this activity.

\section{REFERENCES}

[1] C. Tai and T.-C. Tsao. Control of an electromechanical camless valve actuator. In Proceedings of the 2002 American Control Conference (IEEE Cat. No. CH37301), volume 1, pages 262-267. IEEE, 2002.

[2] Y. Wang, T. Megli, M. Haghgooie, K. S. Peterson, and A.G. Stefanopoulou. Modeling and control of electromechanical valve actuator. Technical report, SAE Technical Paper, 2002.

[3] H. Ito and T. Miyoshi. Electronic fuel injection system for an internal combustion engine having electromagnetic valves and a fuel damper upstream thereof, 1980. US Patent 4,205,637.

[4] Y. Wang. Modeling of an electromechanical valve actuator for a camless engine. In Proc. of AVEC2000 5th Int'1 Symposium on Advanced Vehicle Control, Ann Arbor, USA, pages 22-24, 2000.

[5] P. Tatiossian, F. Dugue, and F. Roux. Electronic pressure regulator for liquid propulsion rockets. Space Propulsion, may 2016. Rome, Italie.

[6] M. H.A. Yaseen. A comparative study of stabilizing control of a planer electromagnetic levitation using pid and lqr controllers. Results in physics, 7:4379-4387, 2017.

[7] S. Di Cairano, A. Bemporad, I. Kolmanovsky, and D. Hrovat. Model predictive control of magnetic automotive actuators. In American Control Conference, 2007. ACC'07, pages 5082-5087. IEEE, 2007.

[8] A. Forrai, T. Ueda, and T. Yumura. Electromagnetic actuator control: A linear parameter-varying (lpv) approach. IEEE Transactions on Industrial Electronics, 54(3):1430-1441, 2007.

[9] C. R. Koch, A. F. Lynch, and R. R. Chladny. Modeling and control of solenoid valves for internal combustion engines. IFAC Proceedings Volumes, 35(2):197-202, 2002.

[10] T. Nguyen, J. Leavitt, F. Jabbari, and J. E. Bobrow. Accurate slidingmode control of pneumatic systems using low-cost solenoid valves. IEEE/ASME Transactions on mechatronics, 12(2):216-219, 2007.

[11] N.F. Al-Muthairi and M. Zribi. Sliding mode control of a magnetic levitation system. Mathematical Problems in Engineering, 2004(2):93107, 2004.

[12] A. Goel and A. Swarup. A novel high-order sliding mode control of magnetic levitation system. In 2016 IEEE 59th International Midwest Symposium on Circuits and Systems (MWSCAS), pages 1-4. IEEE, 2016.

[13] P. Mercorelli. An antisaturating adaptive preaction and a slide surface to achieve soft landing control for electromagnetic actuators. IEEE/ASME Transactions on Mechatronics, 17(1):76-85, 2012.

[14] K. S. Peterson, J. W. Grizzle, and A. G. Stefanopoulou. Nonlinear control for magnetic levitation of automotive engine vales. IEEE Transactions on Control Systems Technology, 14(2):346-354, 2006.

[15] Z. Rao and G. M. Bone. Nonlinear modeling and control of servo pneumatic actuators. IEEE transactions on control systems technology, 16(3):562-569, 2008.

[16] K. S. Peterson and A. G. Stefanopoulou. Extremum seeking control for soft landing of an electromechanical valve actuator. Automatica, 40(6):1063-1069, 2004.

[17] Plecs blockset edition info@plexim.com www.plexim.com.

[18] Comsol multiphysics v5.3 www.comsol.com, comsol ab, stockholm.

[19] T. Schwarzgruber, H. Trogmann, T. E. Passenbrunner, S. Fizek, and P. Dolovai. Nonlinear control of an electro-magnetic actuator under highly dynamic disturbances. In Control Applications (CCA), 2012 IEEE International Conference on, pages 974-979. IEEE, 2012.

[20] F. Deschaux, F. Gouaisbaut, and Y. Ariba. Nonlinear control for an uncertain electromagnetic actuator. In 2018 IEEE Conference on Decision and Control (CDC), pages 2316-2321. IEEE, 2018.

[21] S.-Q. Lee and D.-G. Gweon. A new 3-dof z-tilts micropositioning system using electromagnetic actuators and air bearings. Precision Engineering, 24(1):24-31, 2000

[22] H. Woodson and J. R. Melcher. Electromechanical dynamics. Wiley, 1968

[23] J. Löfberg. Yalmip : A toolbox for modeling and optimization in matlab. In In Proceedings of the CACSD Conference, Taipei, Taiwan, 2004. 
[24] K.-C. Toh, M. J. Todd, and R .H. Tütüncü. Sdpt3a matlab software package for semidefinite programming, version 1.3. Optimization methods and software, 11(1-4):545-581, 1999.

[25] R. Goebel, R. G. Sanfelice, and A. R. Teel. Hybrid dynamical systems. IEEE Control Systems Magazine, 29(2):28-93, 2009. 